\title{
Avaliação do perfil de usuários de hipolipemiantes de uma drogaria em Porto Alegre - RS
}

\author{
Fernanda Maciel Rodrigues* Editiane Alcântara Petruzellis* Patricia \\ Martins Bock ***
}

Resumo: As doenças cardiovasculares (DCV) relacionadas com a dislipidemia,são responsáveis por uma grande taxa de mortalidade e morbidade mundial. $\mathrm{O}$ objetivo deste trabalho foi avaliar o perfil de usuários de hipolipemiantes de uma drogaria de Porto Alegre, bem como o conhecimento desses pacientes sobre a dislipidemia, buscando verificar se a população em estudo realiza adequadamente seu tratamento. 0 estudo apresentou delineamento observacional transversal, a partir de um levantamento epidemiológico e análise de dados qualitativos e quantitativos, entre 97 usuários de hipolipemiantes de uma drogaria em Porto Alegre - RS. A coleta de dados foi obtida por meio de entrevista estruturada, juntamente com a análise de um exame do perfil lipídico de cada paciente. Observou-se que mais da metade dos entrevistados já decidiram parar com seu tratamento hipolipemiante, não fazem acompanhamento médico frequente e se encontram com pelo menos um parâmetro de seu perfil lipídico alterado, sendo os níveis de $\mathrm{HDL}$ colesterol os que mais alterações apresentaram. É de extrema importância a correta orientação ao usuário de hipolipemiante acerca de seu tratamento, por meio de planos baseados na atenção farmacêutica, visando aumentar a adesão ao tratamento hipolipemiante. A utilização correta de hipolipemiantes poderia otimizar os resultados do tratamento.

Descritores: Dislipidemias; Doenças Cardiovasculares; Hipolipemiantes.

\section{Profile evaluation of users of lipid lowering drugs in a drug store of Porto}

Alegre - RS

Abstract: Cardiovascular diseases (CVD) related to dyslipidemia are responsible for a high rate of mortality and morbidity worldwide. The aim of this work was to evaluate the profile of users of a lipidlowering drug store in Porto Alegre, as well the patients knowledge about dyslipidemia, searching if the study population adequately performs its treatment. This cross-sectional observational study design presented from an epidemiological survey and analysis of qualitative and quantitative data among users of a lipid-lowering drug store in Porto Alegre - RS. Data collection was obtained through structured interview, along with analysis of an examination of the lipid profile of each patient. We found that more than half of the respondents have already decided to stop its lipid-lowering therapy, are not frequent medical supervision and show at least one parameter altered lipid profile, $\mathrm{HDL}$ cholesterol showing the most changes. It is extremely important the correct orientation to the user about their lipid-lowering treatment, through plans based pharmaceutical care, to increase adherence to lipid-lowering therapy. The correct use of lipid-lowering could optimize treatment outcomes.

Descriptors: Dyslipidemia, Cardiovascular Disease, Lipid Lowering.

\footnotetext{
* Graduada em Farmácia pelo Centro Universitário Metodista (IPA), Porto Alegre, RS, Brasil.

**Doutoranda em Ciências Biológicas na Universidade Federal do Rio Grande do SUI (UFRGS), Porto Alegre, Brasil.
} 


\section{Introdução}

Dislipidemias são alterações metabólicas lipídicas que levam a um desvio anormal no valor de uma ou mais frações lipídicas séricas. ${ }^{1}$ Podem ser classificadas segundo os padrões das alterações dos níveis de lipoproteínas, através da classificação fenotípica de FredricksonLevy $^{2}$, e participam ativamente no processo aterosclerótico, surgindo como um dos mais importantes fatores de risco no desenvolvimento de Doenças Cardiovasculares (DCV), sendo a principal delas a Doença Arterial Coronariana (DAC). ${ }^{3,4}$

Os riscos de se desenvolver DCV aumentam gradativamente com a elevação dos níveis de colesterol total (CT), LDL colesterol (LDL-C), triglicerídeos (TG) e quilomícrons. ${ }^{1,5,6}$ As DCV são consideradas a principal causa de morte no mundo ${ }^{7}$, assim, a detecção precoce das alterações lipídicas pode contribuir de forma efetiva para evitar o desenvolvimento prematuro da aterosclerose. ${ }^{8}$

A aterosclerose é uma doença inflamatória crônica, que leva a formação de placas lipídicas no lúmen dos vasos sanguíneos. É uma doença progressiva, associada a múltiplos fatores de risco cardiovascular, envolvendo principalmente disfunção endotelial, inflamação e estresse oxidativo. ${ }^{1,9,10,11}$

0 tratamento das dislipidemias visa fundamentalmente à prevenção das doenças ateroscleróticas, por meio da redução do perfil global de risco cardiovascular. Este perfil irá condicionar o nível de intervenção do tratamento, que pode ser não farmacológico ou farmacológico ${ }^{8,12} 0$ tratamento não farmacológico baseia-se em modificações do estilo de vida, através da adoção de hábitos alimentares saudáveis, associados com a prática de exercícios físicos. ${ }^{3}$ Já a terapia farmacológica envolve a utilização de fármacos hipolipemiantes, capazes de isoladamente ou em associação produzir adequação do perfil lipídico. ${ }^{13}$

A farmacoterapêutica para os quadros de dislipidemia baseia-se no uso regular de classes de fármacos hipolipemiantes como resinas sequestrantes de ácidos biliares, fibratos, ácido nicotínico, inibidores seletivos da absorção de colesterol e as estatinas, escolha mais efetiva no tratamento das dislipidemias, tendo ação também sobre a progressão da placa de ateroma, devido ao seu efeito anti-inflamatório. 11, 12, 14, 15

Embora a terapia farmacoterapêutica para redução de colesterol tenha se tornado um grande avanço no tratamento das DCV, a forma com que cada indivíduo conduz seu tratamento pode limitar o benefício alcançado. A falta de adesão ao tratamento contribui para a evolução da doença, sendo precursora da descontinuação do tratamento. Desta forma, 0 alcance dos objetivos terapêuticos se torna prejudicado, aumentando o risco de se desenvolver eventos cardiovasculares. ${ }^{14,16,17}$

O desenvolvimento de planos baseados no seguimento farmacoterapêutico e na atenção farmacêutica, se tornam uma alternativa para otimizar a adesão e o resultado dos tratamentos, com objetivo de reduzir de forma significativa a mortalidade. ${ }^{14,16,18} \mathrm{Assim}$, este trabalho teve por objetivo avaliar o perfil de usuários de hipolipemiantes de uma drogaria de Porto Alegre, bem como o conhecimento desses pacientes sobre a dislipidemia, buscando verificar se a população em estudo realiza adequadamente seu tratamento, para que através dos resultados deste estudo a atenção farmacêutica possa ser melhor aplicada, auxiliando na adesão e correto tratamento hipolipemiante destes pacientes.

Saúde (Santa Maria), v.39, n.2, p.149-160, 2013. Avaliação do perfil dos usuários de hipolipemantes de uma drogaria em Porto Alegre - RS 


\section{Metodologia}

O estudo apresentou delineamento observacional transversal, a partir de um levantamento epidemiológico e análise de dados qualitativos e quantitativos. Foi realizado no período de Janeiro a Maio de 2012, na cidade de Porto Alegre, RS. A população de estudo foi composta por clientes atendidos em uma Drogaria de Porto Alegre, que faziam uso de qualquer medicamento hipolipemiante.

Foram considerados como critérios de inclusão: ser cliente da Drogaria; ambos os sexos; ter idade igual ou superior a 18 anos; estar fazendo uso de qualquer tratamento hipolipemiante, no período do estudo; entender e concordar com os objetivos e metodologia do estudo, aceitando fazer parte do mesmo perante assinatura do termo de consentimento livre e esclarecido. Foram excluídos da pesquisa menores de 18 anos, gestantes, que não faziam uso de medicamentos hipolipemiantes, que apresentassem algum problema cognitivo que impedisse o entendimento do questionário da pesquisa, ou que não aceitassem fazer parte do estudo.

O estudo foi aprovado pelo Comitê de Ética do Centro Universitário Metodista, do IPA (Protocolo n²68/2011). A coleta de dados foi precedida da assinatura do Termo de Consentimento Livre e Esclarecido, (TCLE), para aplicação da entrevista referente à pesquisa. Este termo foi assinado pelo pesquisador responsável, no qual este se comprometeu com o anonimato dos pacientes e o uso dos dados somente para o previsto estudo. Todo o processo de pesquisa obedeceu aos princípios éticos, garantindo aos participantes o consentimento livre e esclarecido, sigilo das informações e privacidade.

Foi realizada uma amostra de conveniência, sendo calculada uma amostra de 97 indivíduos, para se obter um nível de confiança de 95\%, e um erro máximo de 10\%.

A coleta de dados foi obtida por meio de questionário estruturado, abordando características do paciente, informações sobre sua doença, tratamento e problemas relacionados aos medicamentos, juntamente com a análise de um exame do perfil lipídico de cada paciente, o qual era recolhido um dia após a entrevista. A avaliação do perfil lipídico foi caracterizada por valores de referência do Colesterol Total (CT), Triglicerídeos (TG), LDL e HDL colesterol, a partir dos parâmetros considerados desejáveis, limítrofes e alterados. Os valores referendados foram: para CT desejável (<200), limítrofe (200-240) e alterado (>240), para TG, desejável (<200), limítrofe (200-499) e alterado (>400), para LDL-c, desejável (<130), limítrofe (130-160) e alterado (>160) e para HDL-c os valores de referências foram diferenciados pelo gênero, onde, para o gênero masculino foram considerados os parâmetros, desejável $(\geq 55)$, alterado $(<55)$ e para o gênero feminino, desejável $(\geq 65)$, alterado $(<65) .{ }^{19}$

No período de construção e aplicação do instrumento da pesquisa, inexistiam roteiros de entrevistas já validados que avaliassem o perfil de usuários de hipolipemiantes. Assim, o instrumento foi elaborado através de avaliação de artigos, estudos originais que continham questionários que avaliassem a adesão e conhecimento quanto ao tratamento de doenças diversas. Durante a fase de testagem, 0 instrumento de pesquisa foi avaliado por meio de sua aplicação em sete entrevistas, para que pudéssemos avaliar como estes indivíduos interpretavam cada item do instrumento, de forma a desenvolver um questionário facilmente compreendido por indivíduos de diferentes níveis socioculturais. Após esta fase, 0 questionário foi aplicado para toda a amostra do estudo.

Rev. Saúde (Santa Maria), Santa Maria, v.39, n.2, p.149-160 Jul./Dez.2013. Rodrigues, F. M.; Petruzellis, E. A.; Bock, P. M. 
Os dados foram tabulados em um banco de dados utilizando o programa Excel 2003. A análise descritiva dos dados foi feita pela apresentação dos resultados em frequências (variáveis qualitativas), média e desvio padrão (variáveis quantitativas). Foi utilizado para tal análise o software SPSS (Statistical Package for Social Sciences).

\section{Resultados}

O estudo avaliou 97 indivíduos, onde $56,7 \%$ eram do gênero feminino, com idade média de 48,0 $\pm 12,3$ anos, sendo o ensino médio o nível de instrução prevalente (46,3\%). A renda familiar entre 3 e 4 salários mínimos foi a renda de $53,6 \%$ dos entrevistados (tabela 1).

Verificou-se que $(49,5 \%)$ encontrava-se com sobrepeso, que 62,9\% ingeriam alimentos gordurosos todos os dias e $50,5 \%$ consumiam verduras todos os dias, cerca de metade $(52,6 \%)$ raramente ingeriam frutas, 56,7\% não apresentavam restrição de sal e $64,9 \%$ não ingeriam bebidas alcoólicas. Mais da metade dos participantes eram indivíduos fumantes $(59,8 \%)$ e sedentários $(73,2 \%)$. Os indivíduos com familiares que apresentavam dislipidemia constituíram $72,2 \%$ da amostra, tendo a maior parte desta população nunca apresentado nenhuma das doenças vasculares citadas no roteiro da entrevista $(81,4 \%)$. A descrição completa das características do perfil dos entrevistados encontra-se na Tabela1.

\begin{tabular}{|c|c|c|}
\hline Variáveis & $\begin{array}{r}\text { Frequêencia } \\
\text { absoluta (n) }\end{array}$ & $\begin{array}{l}\text { Frequência } \\
\text { relativa (\%) }\end{array}$ \\
\hline \multicolumn{3}{|l|}{ GÊNERO } \\
\hline Masculino & 42 & 43,3 \\
\hline Feminino & 55 & 56,7 \\
\hline \multicolumn{3}{|l|}{ IDADE / ANOS } \\
\hline \multicolumn{3}{|l|}{ Média: $48,0 \pm 12,3$} \\
\hline 15 a 30 & 7 & 7,2 \\
\hline 31 a 45 & 36 & 37,1 \\
\hline 46 a 60 & 38 & 39,2 \\
\hline$>61$ & 16 & 16,5 \\
\hline \multicolumn{3}{|l|}{ ESCOLARIDADE } \\
\hline Analfabeto & 1 & 1,0 \\
\hline Ens. Fund. Completo e incompleto & 28 & 28,9 \\
\hline Ens. Médio completo e incompleto & 45 & 46,3 \\
\hline Ens. Sup. Completo e incompleto & 23 & 23,7 \\
\hline
\end{tabular}




\begin{tabular}{|c|c|c|}
\hline \multicolumn{3}{|l|}{ RENDA FAMILIAR } \\
\hline$<2$ salários & 42 & 43,3 \\
\hline 3 a 4 salários & 52 & 53,6 \\
\hline $5 \mathrm{~A} 10$ salários & 3 & 3,1 \\
\hline \multicolumn{3}{|l|}{ IMC } \\
\hline Peso normal & 15 & 15,5 \\
\hline Sobre peso & 48 & 49,5 \\
\hline Obesidade & 34 & 35,0 \\
\hline \multicolumn{3}{|c|}{ INGERE ALIMENTOS GORDUROSOS } \\
\hline Todos os dias & 61 & 62,9 \\
\hline Raramente & 35 & 36,1 \\
\hline Não ingere & 1 & 1,0 \\
\hline \multicolumn{3}{|l|}{ INGERE FRUTAS } \\
\hline Todos os dias & 45 & 46,4 \\
\hline Raramente & 51 & 52,6 \\
\hline Não ingere & 1 & 1,0 \\
\hline \multicolumn{3}{|l|}{ INGERE VERDURAS } \\
\hline Todos os dias & 49 & 50,5 \\
\hline Raramente & 46 & 47,4 \\
\hline Não ingere & 2 & 2,1 \\
\hline \multicolumn{3}{|c|}{ APRESENTA RESTRIÇÃO DE SAL } \\
\hline Sim & 42 & 43,3 \\
\hline Não & 55 & 56,7 \\
\hline \multicolumn{3}{|c|}{ FAZ ATIVIDADE FÍSICA FREQUÊNTE } \\
\hline Sim & 26 & 26,8 \\
\hline Não & 71 & 73,2 \\
\hline \multicolumn{3}{|l|}{ FUMA } \\
\hline $\operatorname{Sim}$ & 58 & 59,8 \\
\hline Não & 39 & 40,2 \\
\hline \multicolumn{3}{|l|}{ INGERE BEBIDAALCOÓLICA } \\
\hline Sim, até $2 x$ por semana & 15 & 15,5 \\
\hline Sim, $>3 \times$ por semana & 19 & 19,6 \\
\hline Não & 63 & 64,9 \\
\hline
\end{tabular}


TEM FAMILIAR COM DISLIPIDEMIA

\begin{tabular}{ccc} 
Sim & 70 & 72,2 \\
Não & 27 & 27,8 \\
\hline JÁ APRESENTOU ALGUMA DOENÇA VASCULAR & & \\
Isquemia & 15 & 15,5 \\
Infarto agudo do miocárdio & 3 & 3,1 \\
Nenhuma delas & 79 & 81,4 \\
\hline
\end{tabular}

As respostas relacionadas com a adesão ao tratamento hipolipemiante foram examinadas individualmente e encontram-se descritas na Tabela 2. Observou-se que uma elevada parte dos participantes $(61,9 \%)$ acredita que a dislipidemia apresenta consequências graves como o AVC. A maioria dos indivíduos (67,0\%) está em tratamento entre 1 e 5 anos, sendo que $97,9 \%$ estão melhorando com o tratamento realizado.

Tabela 2 - Análise do conhecimento e da adesão dos pacientes ao tratamento hipolipemiante.

\begin{tabular}{|c|c|c|}
\hline Variáveis & $\begin{array}{l}\text { Freqüência } \\
\text { absoluta (n) }\end{array}$ & $\begin{array}{l}\text { Frequência } \\
\text { relativa (\%) }\end{array}$ \\
\hline \multicolumn{3}{|c|}{ CONSEQUÊNCIAS DA DISLIPIDEMIA } \\
\hline Não há consequências & 7 & 7,2 \\
\hline Grave como AVC & 60 & 61,9 \\
\hline Leve como tontura & 14 & 14,4 \\
\hline Não tenho conhecimento & 16 & 16,5 \\
\hline TEMPO DE TRATAMENTO & $\mathrm{n}$ & $\%$ \\
\hline 1 a 5 anos & 65 & 67,0 \\
\hline 6 a 10 anos & 26 & 26,8 \\
\hline$>11$ anos & 6 & 6,2 \\
\hline \multicolumn{3}{|c|}{ TEM MELHORADO COM O TRATAMENTO } \\
\hline Sim & 95 & 97,9 \\
\hline Não & 2 & 2,1 \\
\hline \multicolumn{3}{|c|}{ ALGUMA VEZ PAROU COM O HIPOLIPEMIANTE } \\
\hline Sim & 56 & 57,7 \\
\hline Não & 41 & 42,3 \\
\hline \multicolumn{3}{|c|}{ ESQUECE DE TOMAR SEU HIPOLIPEMIANTE } \\
\hline Sim & 28 & 28,9 \\
\hline Não & 37 & 38,1 \\
\hline As vezes & 32 & 33,0 \\
\hline
\end{tabular}


É CUIDADOSO COM HORÁRIO DE SEU HIPOLIPEMIANTE

\begin{tabular}{ccc} 
Sim & 42 & 43,3 \\
Não & 21 & 21,6 \\
As vezes & 34 & 35,1 \\
\hline PROVIDENCIA MEDICAÇÃO ANTES DE ACABAR & & \\
Sim & 89 & 91,8 \\
Não & 8 & 8,2 \\
\hline HOUVE MUDANÇA DE MEDICAMENTO DURANTE O PERIOODO & & \\
DE TRATAMENTO & & \\
Sim & 29 & 29,9 \\
Não & 68 & 70,1 \\
\hline
\end{tabular}

De acordo com os dados obtidos verificou-se que $57,7 \%$ dos indivíduos da amostra alguma vez já parou com seu tratamento hipolipemiante. Observou-se que a menor parte dos entrevistados (38,1\%) não esquece de tomar seu medicamento, $43,3 \%$ são cuidadosos quanto ao horário de uso, 91,8\% providenciam a medicação antes de acabar e 70,1\% durante o período de tratamento não mudaram de medicamento hipolipemiante.

Com a análise dos resultados podemos observar que mais da metade dos entrevistados, $(54,2 \%)$ já decidiu deixar de tomar seu medicamento hipolipemiante, pelo fato de achar estar se sentindo mal devido ao uso da medicação.

Com relação aos serviços e aos profissionais da saúde se verificou que a maior parte da amostra não faz acompanhamento médico frequente, sendo a dificuldade de conseguir consulta o motivo mais prevalente $(30,9 \%)$. A maioria dos indivíduos $(59,8 \%)$ não está satisfeito com atendimento de saúde, não esclarecem suas dúvidas com o médico $(62,9 \%)$, mas são questionados pelo médico quanto ao uso de seu hipolipemiante $(50,5 \%)$, e pelo próprio são orientados a tomar corretamente seus medicamentos $(46,4 \%)$, tendo a maior parte dos entrevistados perfeito entendimento quanto às explicações dos profissionais de saúde $(80,4 \%)$. A descrição completa dos dados encontra-se na tabela 3.

Tabela 3 - Análise do uso dos serviços de saúde e avaliação dos profissionais de saúde.

\begin{tabular}{|c|c|c|}
\hline Variáveis & $\begin{array}{c}\text { Freqüênci } \\
\text { a absoluta } \\
\text { (n) }\end{array}$ & $\begin{array}{c}\text { Frequênci } \\
\text { a relativa } \\
(\%)\end{array}$ \\
\hline \multicolumn{3}{|l|}{ FAZ ACOMPANHAMENTO MÉDICO FREQUENTE } \\
\hline Sim, 1 a 5 vezes ano & 18 & 18,5 \\
\hline Sim, > 6 vezes ano & 12 & 12,4 \\
\hline Não, falta de tempo & 28 & 28,9 \\
\hline Não, difícil cons. Consulta & 30 & 30,9 \\
\hline Não, por esquecimento & 9 & 9,3 \\
\hline
\end{tabular}

Rev. Saúde (Santa Maria), Santa Maria, v.39, n.2, p.149-160 Jul./Dez.2013. Rodrigues, F. M.; Petruzellis, E. A.; Bock, P. M. 
ESTA SATISFEITO COM ATENDIMENTO PRESTADO A SUA SAÚDE

$\operatorname{Sim}$

Não

VOCÊ ESCLARECE SUAS DÚVIDAS COM O MÉDICO

$\begin{array}{lll}\text { Sim } & 36 & 37,1 \\ \text { Não } & 61 & 62,9\end{array}$

\begin{tabular}{ccc}
\hline MÉDICO PERGUNTA SE ESTA TOMANDO CORRETAMENTE SEUS MEDICAMENTOS & \\
Sim & 48 & 49,5 \\
Não & 49 & 50,5 \\
\hline QUEM O ORIENTAA TOMAR SEUS REMÉDIOS & 45 & 46,4 \\
Médico & 4 & 4,1 \\
Enfermeiro & 4 & 22,7 \\
Farmacêutico & 22 & 26,8 \\
Outros & 26 & \\
\hline ENTENDE AS EXPLICAÇÕES DOS PROFISSIONAIS DE SAÚDE & & 80,4 \\
Sim & 78 & 19,6 \\
Não & 19 &
\end{tabular}

Com relação ao tratamento farmacoterapêutico, se pode analisar que houve a utilização de duas classes de medicamentos hipolipemiantes, estatinas e fibratos. As estatinas foram prescritas na maior parte dos casos $(73,2 \%)$ ao contrário dos fibratos que foram prescritos na minoria dos casos $(26,8 \%)$.

Analisando o perfil lipídico de toda a população de estudo verificou-se que a maioria dos entrevistados parecia encontrar-se com os níveis de CT aumentados (51,5\%), LDL-C na categoria considerada limítrofe $(55,7 \%)$ e os TG na categoria considerada desejável $(38,1 \%)$. Com relação ao $\mathrm{HDL}$ colesterol (HDL-c) se pode verificar que a maioria dos indivíduos tanto do gênero feminino quanto do masculino, estava com seus níveis na categoria considerada alterada.

\section{Discussão}

No presente estudo observou-se a prevalência do gênero feminino, sugerindo uma atenção feminina maior nos aspectos de saúde preventiva. 20 Mais da metade dos indivíduos analisados apresentaram faixa etária maior que 45 anos, evento provavelmente ligado ao fato da dislipidemia ter uma maior prevalência em adultos mais velhos. 1,4

A maior parte dos pacientes usuários de hipolipemiantes entrevistados tem uma renda familiar na faixa de 3 a 4 salários mínimos, faixa caracterizada como classe média $D$, com

Saúde (Santa Maria), v.39, n.2, p.149-160, 2013. Avaliação do perfil dos usuários de hipolipemantes de uma drogaria em Porto Alegre - RS ISSN 2236-58347 grau de escolaridade predominante para o ensino médio incompleto. Observa-se uma maior prevalência de doenças crônicas como a dislipidemia em pacientes de baixa renda e baixo nível de escolaridade. ${ }^{21,22}$ 
Durante algum tempo, pensava-se que as doenças cardiovasculares seriam determinadas geneticamente, e que pouco poderia ser feito para preveni-las. Porém, esta ideia foi modificada a partir do momento em que seus fatores de risco foram identificados. ${ }^{8,23}$ Mesmo sendo a dislipidemia um dos principais fatores de risco modificáveis para 0 desenvolvimento de doenças cardiovasculares, podemos observar que a maioria dos indivíduos investigados não mantêm o controle dos principais fatores associados à dislipidemia, pois a maioria dos entrevistados eram tabagistas, não praticavam atividades físicas, estavam com sobrepeso, ou em algum grau de obesidade. Questionados sobre 0 consumo de alimentos gordurosos, referiram ter o costume de ingeri-los todos os dias. Somando-se a isto, a maior parte dos indivíduos relatava ter familiares com dislipidemia. Tais hábitos levam ao emprego de hipolipemiantes geralmente de uso contínuo. ${ }^{1,4}$

O estudo apontou que a maioria dos entrevistados está ciente de que a dislipidemia pode gerar consequências cardiovasculares graves, estando a maior parte em tratamento de 1 a 5 anos, sem mudança de medicamento, e com notada melhora devido ao tratamento realizado. Porém o estudo aponta que mais da metade dos indivíduos já por algum motivo decidiu parar de usar seu medicamento hipolipemiante, tendo a maior parte dos entrevistados, tomado esta decisão devido ao fato de se sentir mal pelo uso da medicação ou devido a estar já se sentindo bem com o uso da própria. Este fato provavelmente está ligado à percepção do paciente acerca da dificuldade, duração, segurança e propósito do tratamento. Juntamente a isto, está provavelmente a falta de informação substancial a respeito de seu tratamento, favorecendo assim a sua não adesão e ainda estimulando-o a terapêuticas não convencionais, assim é de suma importância a orientação ao usuário de hipolipemiante. ${ }^{24}$

Em relação aos serviços de saúde verificou-se que $69,1 \%$ dos entrevistados não fazem acompanhamento médico frequentemente, sendo a dificuldade de conseguir consulta 0 motivo mais prevalente. A maioria dos pacientes não está satisfeito com 0 atendimento prestado a sua saúde e não esclarecem suas dúvidas com o médico, apesar de entenderem as explicações dos profissionais de saúde. Segundo a Organização Mundial da Saúde, a adesão ao tratamento avalia o grau de concordância entre o comportamento de um indivíduo em relação às recomendações do profissional da saúde no que se refere ao uso de medicamentos e a mudanças no estilo de vida. Desta forma os fatos estabelecidos por este estudo provavelmente indicam um tratamento com efetividade reduzida, pela falta de informação dos pacientes, pois o termo adesão refere-se à participação ativa do paciente, havendo, por parte deste, o entendimento, esclarecimento e concordância com o tratamento, perante o profissional de saúde. ${ }^{16,25}$

0 estudo mostra que metade dos indivíduos da amostra eram questionados e orientados pelo médico e não pelo farmacêutico quanto à utilização de seu hipolipemiante. Este fato provavelmente se dá devido ao baixo índice de atenção farmacêutica, o que deve ser modificado, pois a mesma tem por objetivos aconselhar e educar, através de informação clara, sobre o bom uso dos medicamentos, incentivando a adesão ao tratamento. ${ }^{26}$

Dentre os medicamentos hipolipemiantes mais prescritos se obteve maior prevalência do uso das estatinas $(73,2 \%)$. Isso se dá provavelmente devido ao fato de que as estatinas fazem parte da classe de hipolipemiantes mais efetiva. Seus efeitos são resultantes da atividade inibidora das estatinas sobre a enzima hidroximetilglutaril-CoA redutase (HMG-CoA Rev. Saúde (Santa Maria), Santa Maria, v.39, n.2, p.149-160 Jul./Dez.2013. Rodrigues, F. M.; Petruzellis, E. A.; Bock, P. M. redutase), a qual bloqueia a con`versão do substrato HMG-CoA em ácido mevalônico, 
inibindo os primeiros passos da biossíntese de colesterol, que leva a regulação dos receptores de $L D L-c$, diminuindo assim os níveis de $L D L$ aterogênicas. ${ }^{27} \mathrm{~A}$ utilização das estatinas está, também, de acordo com as orientações da IV Diretrizes Brasileiras sobre Dislipidemias, que apontam esta classe como uma das principais opções para o tratamento da doença. ${ }^{1}$

Outro aspecto relevante para alta prevalência do uso das estatinas pelos usuários de hipolipemiantes desta drogaria está provavelmente relacionado com 0 fato de 0 estabelecimento dispor do programa Farmácia Popular. O Programa Farmácia Popular do Brasil criado pelo Governo Federal, tem por objetivo ampliar o acesso aos medicamentos para as doenças mais comuns entre os cidadãos. O Programa possui parceria com farmácias e drogarias da rede privada, chamada de "Aqui tem Farmácia Popular", levando o benefício da aquisição de medicamentos e insumos essenciais a baixo custo ou gratuitamente, a mais lugares e a mais pessoas. E dentre os medicamentos dispostos no programa Farmácia Popular encontra-se um tipo de estatina, que é disponibilizada para os clientes da Drogaria com $90 \%$ de desconto. ${ }^{28}$

Analisando o perfil lipídico dos usuários de hipolipemiantes podemos verificar que a maioria dos indivíduos tinha seu perfil lipídico nas categorias limítrofe ou alterada. Além disso, a maioria dos entrevistados encontrava-se com sobrepeso ou em algum grau de obesidade, fato que concordou com outros estudos, que propõe que o excesso de peso pode estar associado a alterações nos níveis de lipídios plasmáticos. ${ }^{29}, 30$

Considerando ambos os gêneros, a maioria dos participantes estava com o HDL-c na categoria alterada. Tal observação provavelmente se relaciona com a relação entre o Índice de Massa Corporal e o perfil lipídico, que pode ser explicada pela ativação da via AMPcquinase dependente, induzida pelo aumento da insulina e da leptina, bem como pela redução da ativação da adiponectina, que leva ao aumento da oxidação dos ácidos graxos. ${ }^{30,31}$

\section{Conclusões}

Os resultados mostraram que as dosagens de CT, TG, LDL-c e HDL-C, tiveram uma maior prevalência nas categorias limitrofe e alteradas, juntamente com uma alta taxa de sobrepeso, obesidade, sedentarismo e interrupção do tratamento.

Este estudo sugere a importância da orientação ao paciente a respeito da dislipidemia e seu tratamento, para que se possa atingir a meta terapêutica gerando perspectivas de implementação de um programa de atenção farmacêutica nesta Drogaria, objetivando, com a participação do farmacêutico, aumentar a adesão ao tratamento hipolipemiante e o incentivo a adoção de hábitos de vida mais saudáveis, de forma a otimizar os resultados dos tratamentos e reduzir a ocorrência de doenças e complicações evitáveis.

\section{Referências Bibliográficas}

Saúde (Santa Maria), v.39, n.2, p.149-160, 2013. Avaliação do perfil dos usuários de hipolipemantes de uma drogaria em Porto Alegre - RS ISSN 2236-58347
1. Sposito AC, Caramelli B, Fonseca FAH, Bertolami M. IV Diretriz Brasileira sobre Dislipidemias e Prevenção da Aterosclerose: Departamento de Aterosclerose da Sociedade Brasileira de Cardiologia. Arq. Bras. Cardiol. 2007; 88(1): 2-19. 
2. Campos EM. Da classificação dos fenotipos de fredrickson - perfis das lipoproteínas - ao entendimento dos genótipos. Acta Med Port. 2004; 18: 189-198.

3. Abadi BL, Budel JM. Aspectos Clínicos Laboratoriais das Dislipidemias. Cadernos da Escola de Saúde, Curitiba. 2011; 1(5): 158-169.

4. Kolankiewicz F, Giovelli FB, Bellinaso ML. Estudo do perfil lipídico e da prevalência de dislipidemias em adultos. Ver. Brás. An. Clin. 2008; 40(4): 317-320.

5. Yuan G, Al-Shali ZK, Hegele AR. Hypertriglyceridemia: its etiology, effects and treatment. Canadian Medical Association or its licensors. 2007; 176(8): 1113-1120.

6. Goldberg IJ, Eckel RH, Mcpherson R. Triglycerides and Heart Disease, Still a Hypothesis? Arterioscler Thromb Vasc Biol. 2011; 31(8): 1716-1725.

7. WHO. Cardiovascular diseases. Key facts. Disponivel em: http://www.who.int/mediacentre/factsheets/fs317/es/index.html. Acesso em: 18 de outubro de 2011.

8. Santos J, Guimaraes A, Diament J. Consenso Brasileiro Sobre Dislipidemias Detecção, Avaliação e Tratamento. Arq. Bras. Endocrinol. Metab. 1999; 43(4): 287-305.

9. Halperin RO, Sesso, HD, Ma J, Buring JE, Stampfer MJ, Gaziano M. Dyslipidemia and risk of incident hypertension in men. Hypert, 2006; 47: 45-50.

10. Camacho CRC, Melicio LAD, Soares AMVC. Aterosclerose, uma resposta inflamatória. Arq. Ciênc. Saúde. 2007; 14(1): 41-48.

11. Mizuno Y, Jacob RF, Mason RP. Inflammation and the Development of Atherosclerosis - Effects of lipidlowering therpy. Journal of Atherosclerosis and Thrombosis. 2011; 18(5): 351-358.

12. Marques SP. Terapêutica redutora intensiva do colesterol: a certeza da segurança. Revista Port Cardiol, 2010; 29(9): 1405-1418.

13. Schulz I. Tratamento das dislipidemias: como e quando indicar a combinação de medicamentos hipolipemiantes. Arq. Bras. Endocrinol Metab. 2006; 50(2): 344-359.

14. Fiegenbaum M, Hutz MH. Farmacogenética de Fármacos Hipolipemiantes. Medicina, Ribeirão Preto, 2006; 39(4): 543-53.

15.Rosendo AB, Dal-Pizzol F, Fiegenbaum M, Almeida S. Farmacogenética e efeito antiinflamatório dos inibidores da HMG-CoA redutase. Arq. Bras. Endocrinol. Metab. 2007; 51(4): 520-525.

16. Osterberg L, Blaschke T. Adherence to medication. N Engl J Med. 2005; 35(5): 487.

17. Morrow DG, Weiner M, Deer MM, Young JM, Dunn S, McGuire P, et al. Patient-centered instructions for medications prescribed for the treatment of Heart failure. Am J Geriatr Pharmacother. 2004; 2: 44-52.

18. Eizerik DP, Manfroi WC. Eficácia da Atenção Farmacêutica em Dislipidemia: Revisão Sistemática. HCPA. 2008; 28(1): 37-40.

19. Motta TV. Bioquímica clínica para laboratório: Princípios e interpretações. $5^{\circ}$ edição. Porto Alegre: Editora Medbook, 2009.

20. Batista MCR, Franceschini SCC. Impacto da atenção nutricional na redução dos níveis de colesterol sérico de pacientes atendidos em serviços públicos de saúde. Arq. Bras. de Cardiol. 2003; 80(2): 162-166.

21. Souza LJ, Souto-Filho JTD, Souza TF, Reis AFF, Gicovate-Neto C, Bastos DA, et al. Prevalência de dislipidemia e fatores de risco em Campos do Goytacazes. Arq. Bras. De Cardiol. 2003; 81(3): 249-256.

22. Gus I, Fischmann A, Medina, C. Prevalência dos fatores de risco da doença arterial coronariana no estado do Rio Grande do Sul. Arq. Bras. de Cardiol. 2002; 78(5): 478-483.

Rev. Saúde (Santa Maria), Santa Maria, v.39, n.2, p.149-160 Jul./Dez.2013. Rodrigues, F. M. Petruzellis, E. A. Bock P.M.

23. Mascarenhas CHM, Reis LA, Souza MS. Avaliação do risco de doença coronariana em adultos e idosos no município de Lagêdo do Tabocal / BA. Arq. Ciênc. Saúde. 2009; 13(1): 15-20. 
24. Mansur AP, Mattar APL, Tsubo CE, Simão DT, Yoshi FR, Daci K. Prescrição e aderência das estatinas com doença arterial coronariana e hipercolesterolemia. Arq Bras Cardiol. 2011; 76: 111.

25. WORLD HEALTH ORGANIZATION, WHO. Adherence to long-term therapies: evidence for action. Geneva: WHO, 2003. Disponivel em: http://www.who.int/whr/2010/whr10_pt.pdf. Acesso em: 23/05/2012.

26. Lee SS, Cheung PY, Chow M.S. Benefits of individualized counseling by the pharmacist on the treatment outcomes of hyperlipidemia in Hong Kong. J Clin Pharmacol. 2004; 44(6): 632-9.

27. Girardi JM, Silva AF, Andrade FC, Rodrigues VB, Raposo NRB. Sub-otimização terapêutica da dislipidemia em portadores de aterosclerose coronariana significativa. HU Revista. 2011; 37(2): 181-189.

28. BRASIL. Portal Saúde. Farmácia Popular. Disponível em: http://portal.saude.gov.br/portal/saude/area.cfm?id_area=1095. Acesso em: 23/05/2012.

29. Dias P, Reis R, Parente F, Silva JM, Moura JJA. Evolução do peso numa consulta de dislipidemias. Medicina Interna. 2005; 12(4): 177-188.

30. Bertoni VM, Zanardo VPS, Ceni GC. Avaliação do perfil lipídico dos pacientes com dislipidemia atendidos no ambulatório de especialidades de nutrição da uricepp. Perspectiva. 2011; 35(129): 177-188.

31. Ramos AT, Carvalho DF, Gonzaga NC, Cardoso AS, Noronha JAF, Cardoso MAA. Perfil lipídico em crianças e adolescentes com excesso de peso. Revista Brasileira de Crescimento Desenvolvimento Humano. 2011; 21(3): 780-788.

\section{Fernanda Maciel Rodrigues}

Endereço para correspondência - Rua Avaí, n. 137, apto. 402. Bairro Centro, CEP: 90050-200, Porto Alegre, RS, Brasil.

Currículo Lattes:

E-mail: nandafmr@gmail.com

Recebido em 21 de novembro de 2012.

Publicado em 05 de novembro de 2013. 